\title{
Skin blood flow measurements during exposures to emotionally charged movies
}

\author{
Samir Henni ${ }^{1} \cdot$ Pierre Abraham ${ }^{1}[0$ \\ Received: 23 February 2018 / Accepted: 13 April 2018 / Published online: 24 April 2018 \\ (c) The Physiological Society of Japan and Springer Japan KK, part of Springer Nature 2018
}

\begin{abstract}
A recent report in the journal evaluated microvascular response at the facial and peripheral level during various types and levels of emotional stresses with laser speckle contrast imaging. Our short letter aims at underlying the importance of artifact removal in this situation and suggest directions for improving the results of this kind of experiment.
\end{abstract}

\section{Dear Editor,}

We read with great interest the paper of Matsukawa et al. [1] evaluating microvascular response at the facial and peripheral level during various types and levels of emotional stresses.

Laser speckle contrast imaging (LSCI) has been proposed for a non-contact measurement of microcirculatory flow in human subjects. LSCI facilitates the routine investigation of cutaneous blood flow (CBF). Unfortunately, as a non-contact technique and as underlined by the authors, LSCI is highly sensitive to movements of the studied area, which result in artifacts superimposing over the CBF signal. Manual removal of obvious high-amplitude artifacts as performed by the authors [1] is not satisfactory because it does only account for ample movements. We previously showed that using an opaque bilayer adhesive patch (OBA) allows automatic observer independent removal of artifacts from Pericam PSI (Perimed; Jarfalla, Sweden) LSCI recordings. The patch includes an aluminum layer to block the laser signal and a polyurethane film. This OBA backscatters a signal to the LSCI device that is completely independent from CBF and linearly proportional to movement artifact observed at the skin level [2,3]. Thereby, subtraction of the OBA signal from the skin signal allows for nonsubjective denoising of LSCI signals even from minor movements [2]. This method improves the reliability of

Pierre Abraham

piabraham@chu-angers.fr

1 Laboratory of Exercise Physiology and Vascular Medicine, University Hospital, 49933 Angers Cedex 09, France
CBF recordings [3]. It would be of interest to confirm the authors' results by repeating their experiment with such an automatic denoising approach.

Further, we showed that not only surface movements but also air movements between the LSCI head and the area to be measured interfere with CBF recordings [4]. This is likely an issue for the "subnasal" area measured by the authors [1]. Possibly at this level, the recording of CBF through LSCI could be gated to breathing movements as can be monitored with chest plethysmography [5] and CBF should be recorded only during the end of the expiratory movement.

Funding No funds were received in support of this work.

\section{Compliance with ethical standards}

Conflict of interest No financial activities outside the submitted work. The manuscript submitted does not contain information about medical devices/drugs.

\section{References}

1. Matsukawa K, Endo K, Ishii K, Ito M, Liang N (2018) Facial skin blood flow responses during exposures to emotionally charged movies. J Physiol Sci. 68(2):175-190

2. Mahe G, Rousseau P, Durand S, Bricq S, Leftheriotis G, Abraham P (2011) Laser speckle contrast imaging accurately measures blood flow over moving skin surfaces. Microvasc Res 81(2):183-188

3. Omarjee L, Signolet I, Humeau-Heutier A, Martin L, Henrion D, Abraham P (2015) Optimisation of movement detection and artifact removal during laser speckle contrast imaging. Microvasc Res 97:75-80 
4. Mahe G, Durand S, Humeau A, Leftheriotis G, Rousseau P, Abraham P (2012) Air movements interfere with laser speckle contrast imaging recordings. Lasers Med Sci 27(5):1073-1076
5. Massaroni C, Carraro E, Vianello A et al (2017) Optoelectronic plethysmography in clinical practice and research: a review. Respiration. 93(5):339-354 